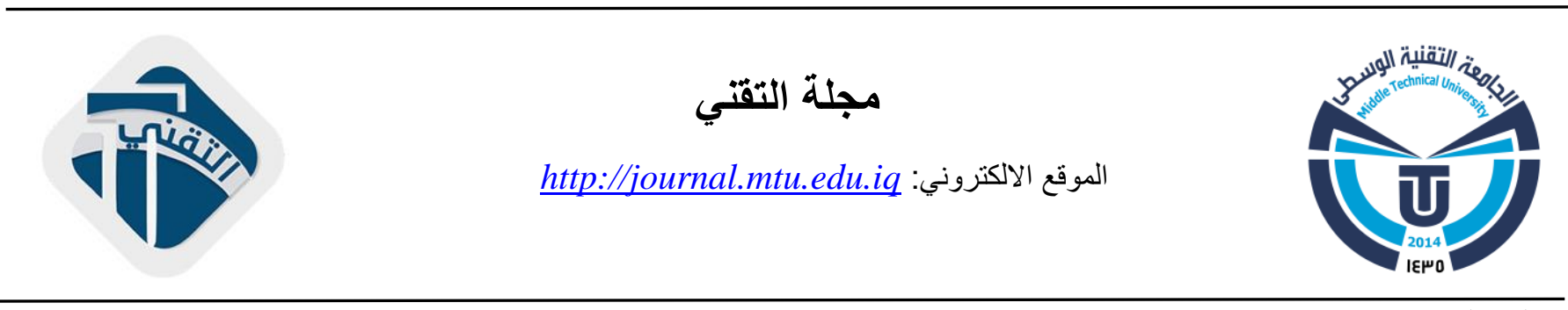

مقالة بحثية

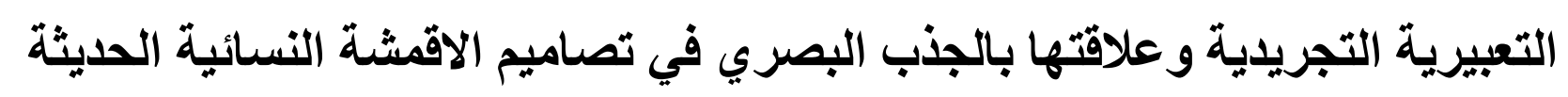

\author{
حيدر هاشم محمود الحسيني \\ معهد الفنون التطبيقية، الجامعة التقنية الوسطى، بغداد، العراق \\ haidar.h.h.h.23@mtu.edu.iq البريد الإلكتروني:
}

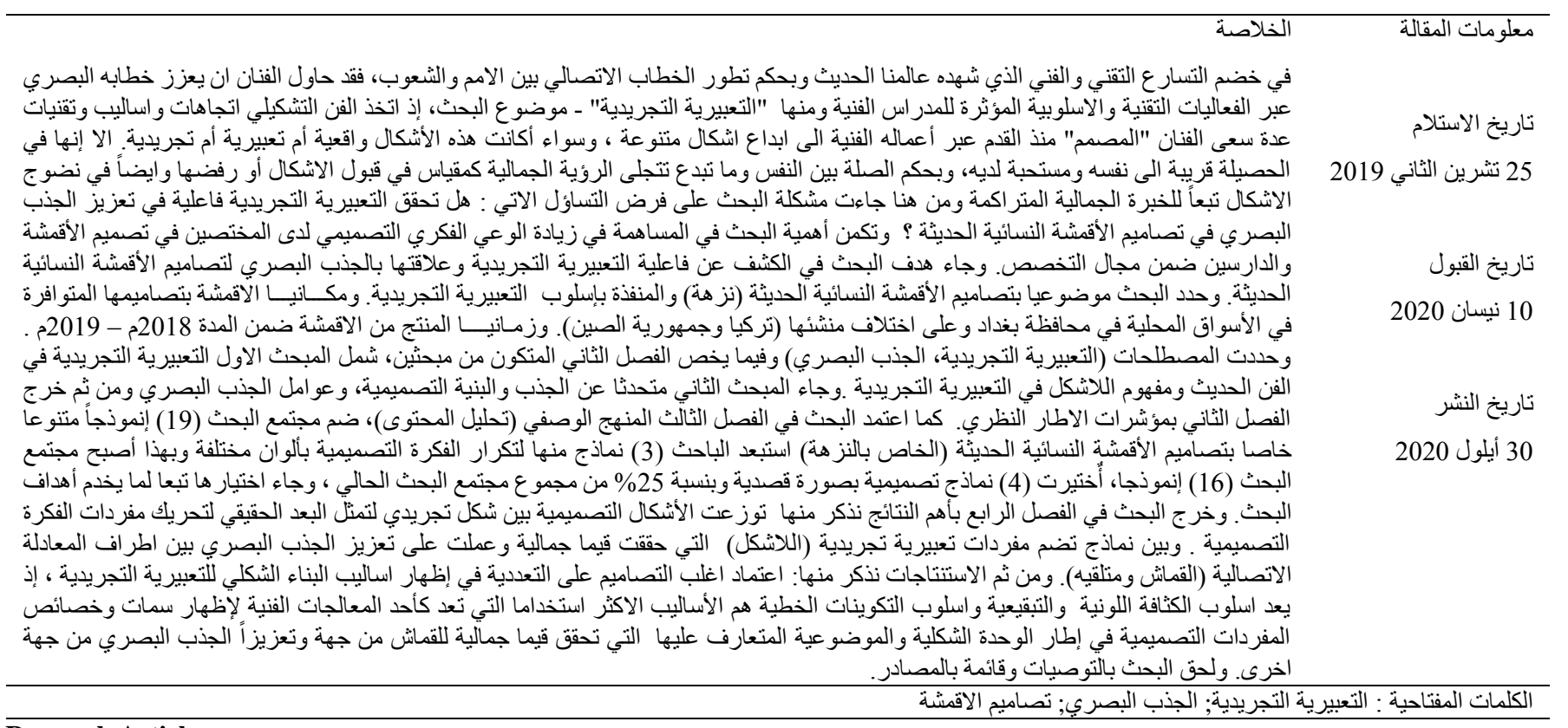

Research Article

\title{
Abstract expressionism and its relationship to visual attraction In the designs of modern women's fabrics
}

\section{Haider Hashim Mammuod Al - Husseini}

Institute of Applied Arts, Middle Technical University, Baghdad, Iraq

*Corresponding author E-mail: haidar.h.h.h.23@mtu.edu.iq

\begin{tabular}{|c|c|}
\hline Article history & Abstract \\
\hline $\begin{array}{l}\text { Received } \\
25 \text { Nonmember } 2019\end{array}$ & $\begin{array}{l}\text { In the midst of the technical and artistic acceleration that we witnessed in our modern world and its development among nations and } \\
\text { people. The artist is trying to amend the visual experiences throughout technical and behavioral artistic approaches among which is } \\
\text { the, "abstract expressionism" - the subject of research, where abstract artist visits several trends, methods and techniques "Designer" } \\
\text { since ancient times through his artwork to create a variety of forms, whether these forms, expressive or abstract. With regard to }\end{array}$ \\
\hline $\begin{array}{l}\text { Accepted } \\
10 \text { April } 2020\end{array}$ & $\begin{array}{l}\text { mixing, loss, complacency or rejection. The designer tries to answer the following questions of: whether abstract expressionism in } \\
\text { the design of modern women's fabrics achieves attraction. A goal is found in the search for modern women's elegance. The research }\end{array}$ \\
\hline
\end{tabular}


Publishing

30 September 2020 objectively identified the designs of modern women's fabrics (picnic) that implemented in the style of abstract expressionism. It also spots for fabrics designs in the local markets in Baghdad which have different countries of origin (Turkey and the Republic of China) for the time period 2018 - 2019. Terminology (abstract expressionism, visual tour) was defined theoretically. As in the third chapter of the descriptive approach (content annexation), the research community included a variety of 19 models for the design of modern women's fabrics (for a picnic) The researcher excluded three models from the repetition of the design idea in different colors. Four design models were chosen intentionally and $25 \%$ of the total current research community. It was selected according to the research objectives. The research came out in the fourth chapter with the most important results where the design forms were divided between an abstract form to represent the real dimension of moving the vocabulary of the design idea. Among the models include abstract expressive vocabulary (non-form), which are aesthetic values and worked to enhance the visual between the sides of the communicative equation (cloth and recipients). The conclusions included: two most common designs rely on pluralism in the formations to cling to the experience, and modify your style of work, and configurations for your staff in the design and qualification in the design and qualification on the one hand and promote attractions and the right to search recommendations and a list of sources.

1 . 1 التعبيرية التجريدية في الفن الحديث 1.1

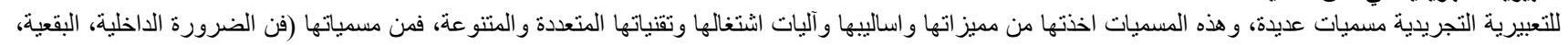

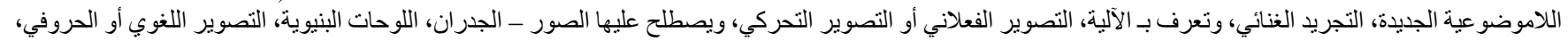

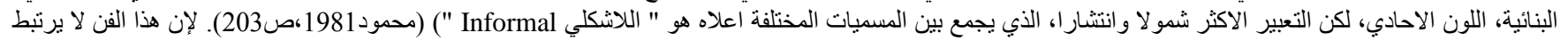

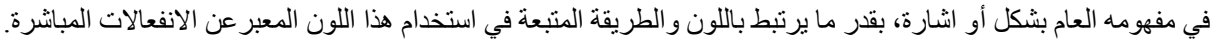

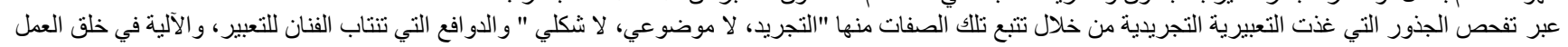

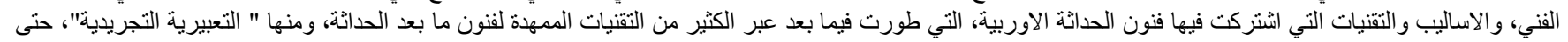

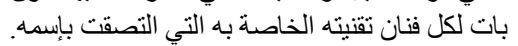

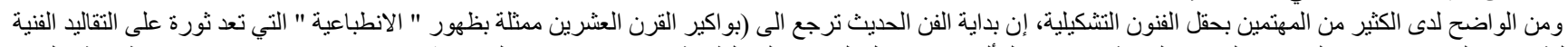

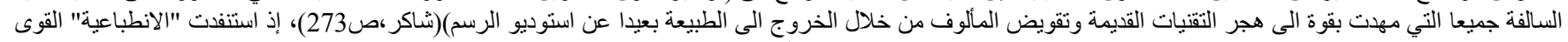

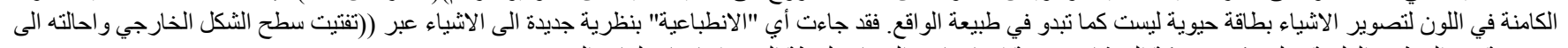

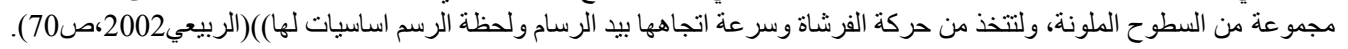

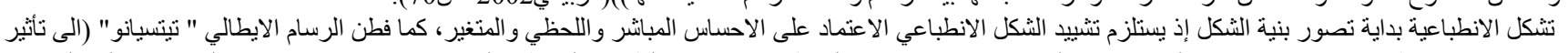

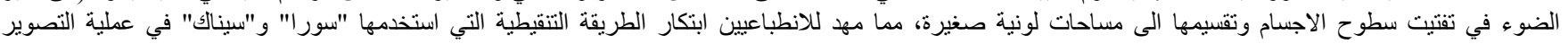

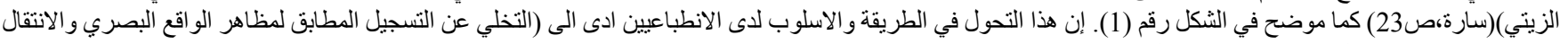

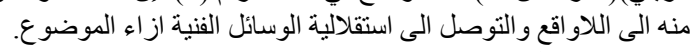

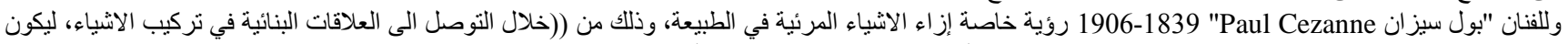

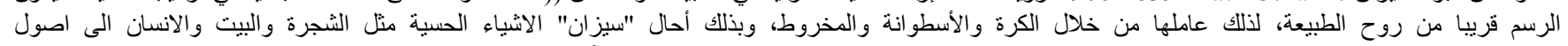

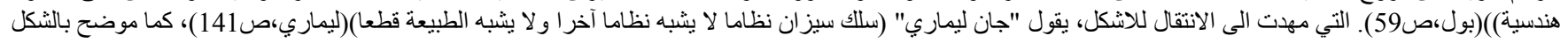

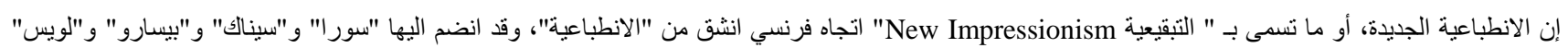

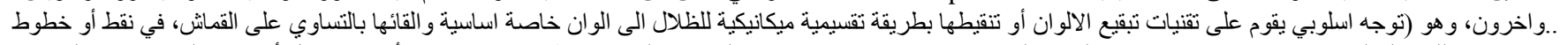

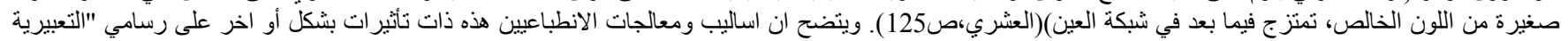
التجريدية" من تجريد وتنقيع ...الخ.

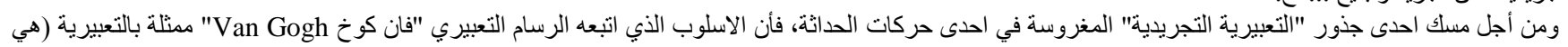

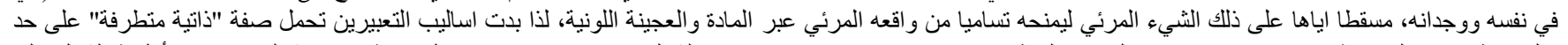

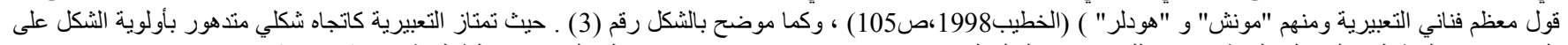

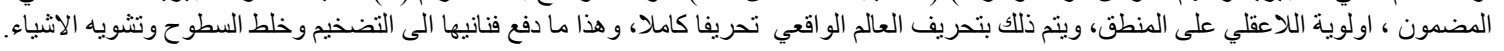

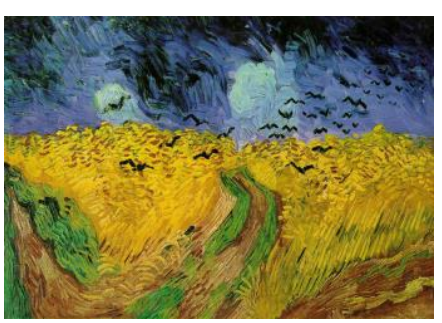

(3) شنكل

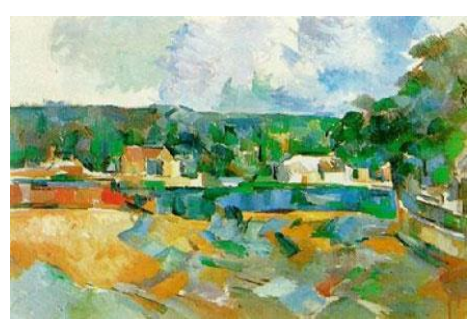

(2) شكل

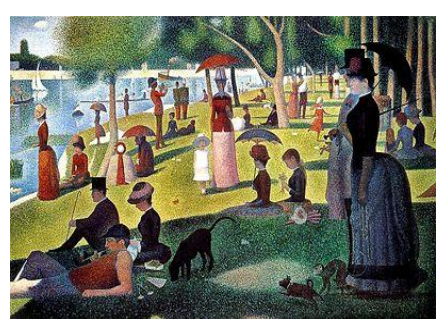

شكل (1)

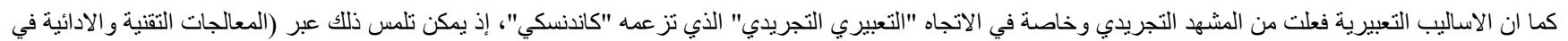

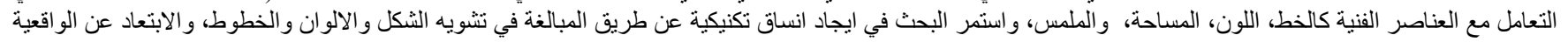

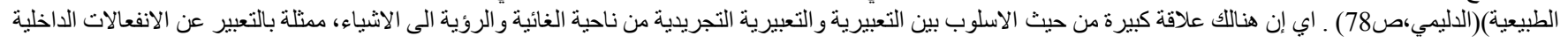

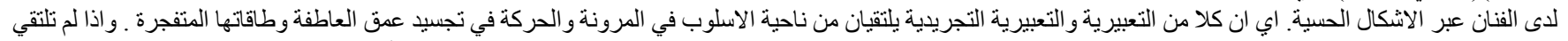

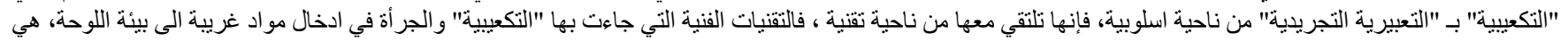




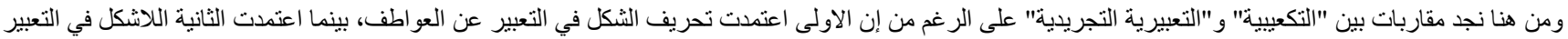

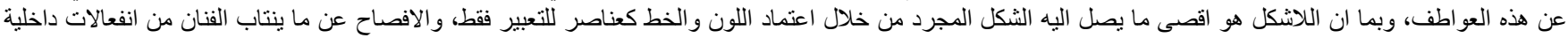

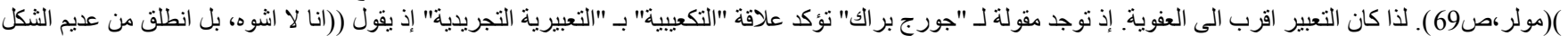

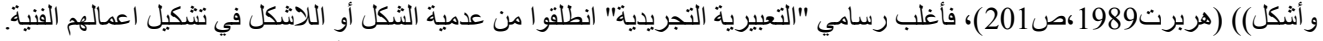

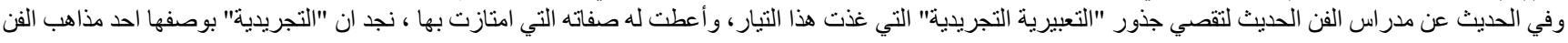

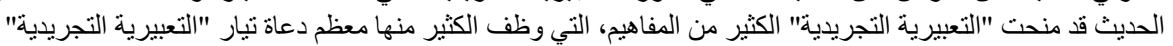

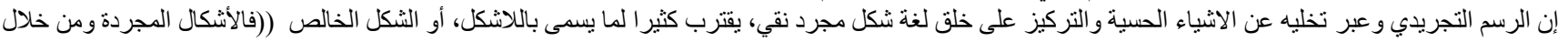

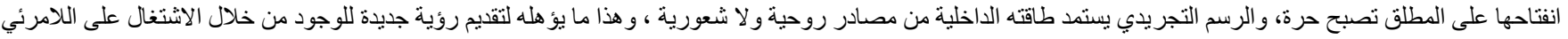

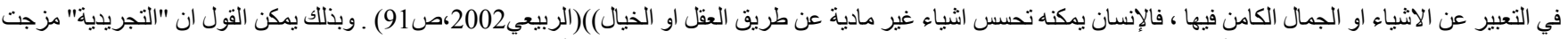

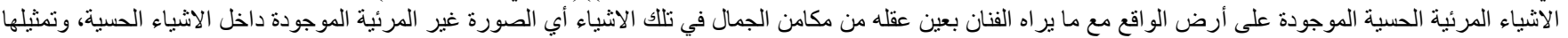

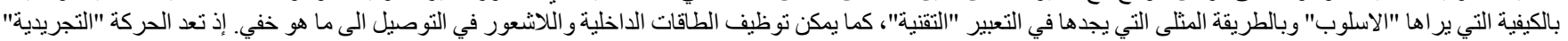

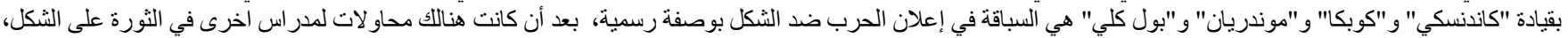

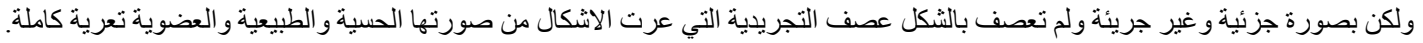

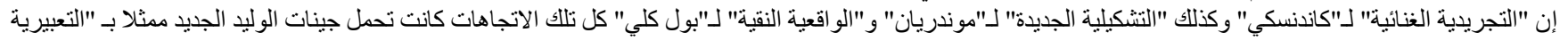

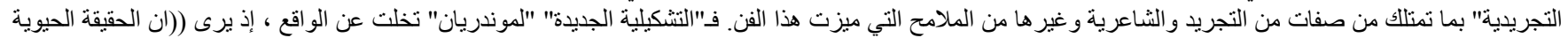

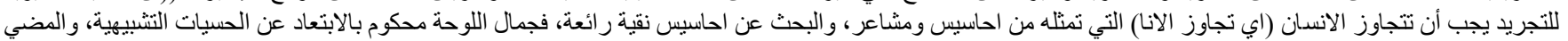

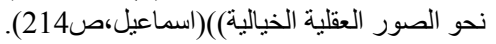

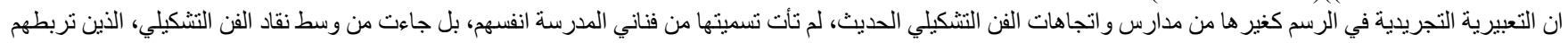

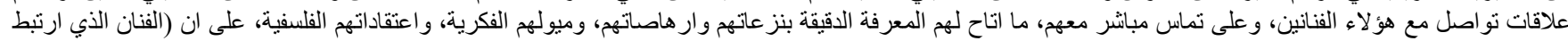

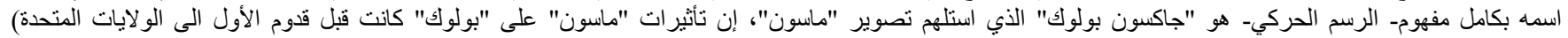

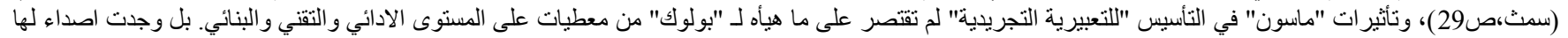

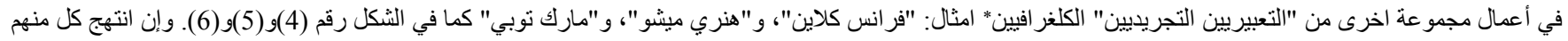

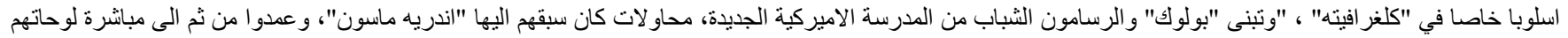

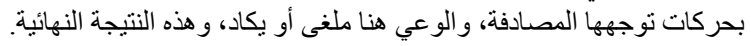

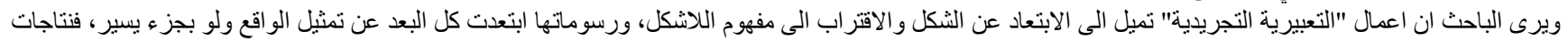

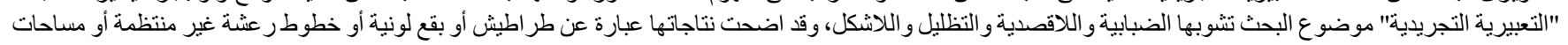

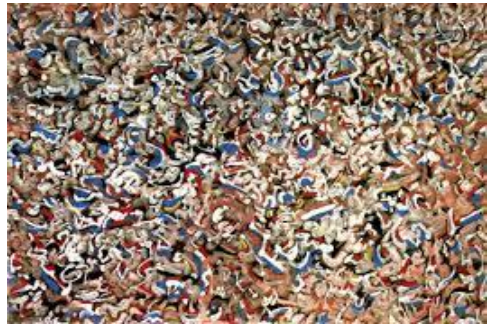

(6) شs

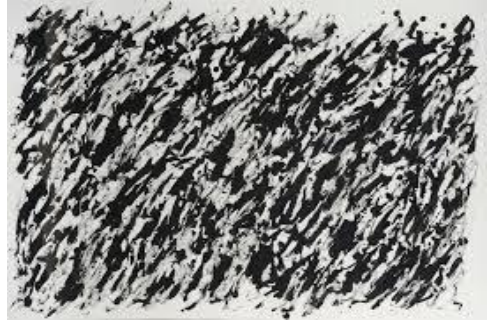

شكل(5)

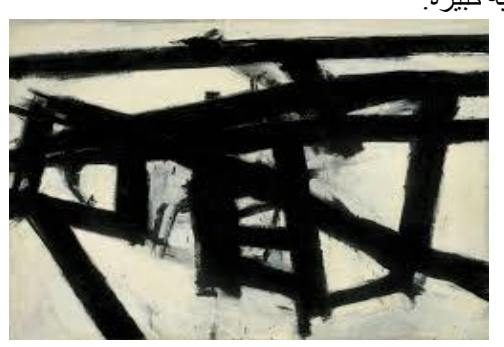

(4)

1.2 مفهوم اللاشكل في التعبيرية التجريدية

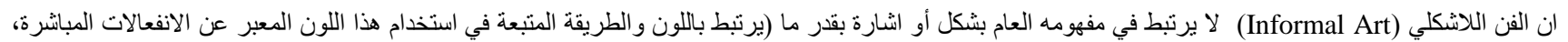

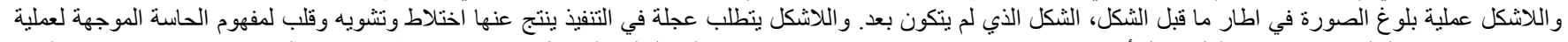

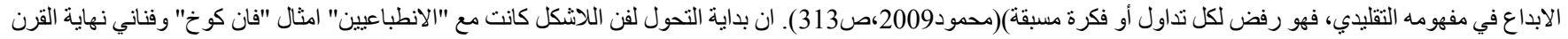

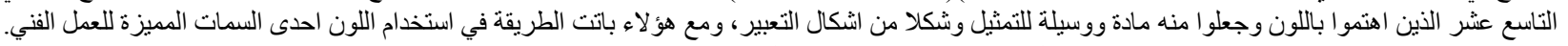

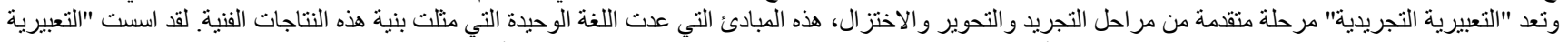

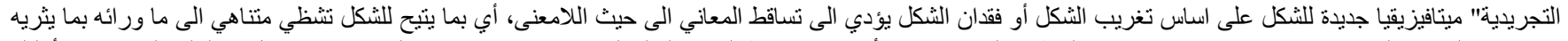

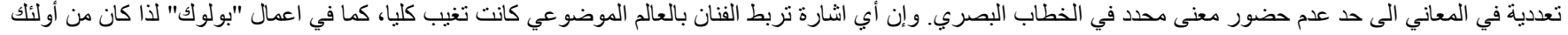

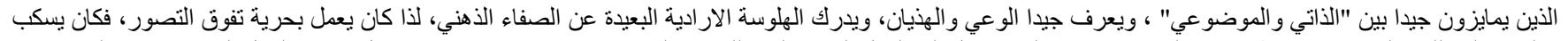

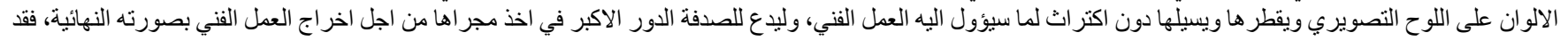

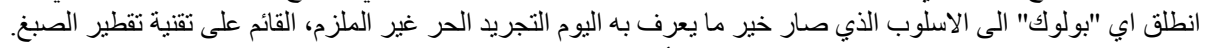

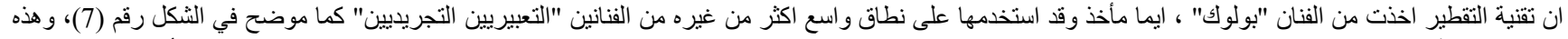

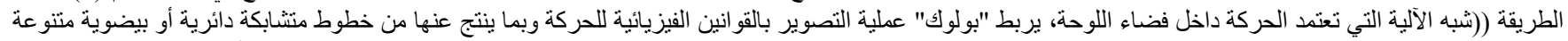

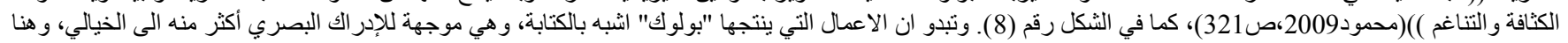

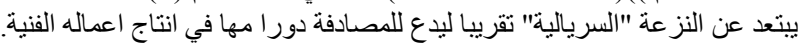

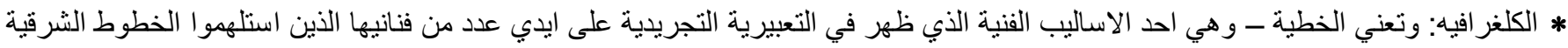

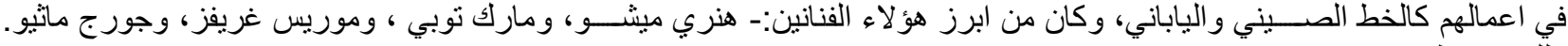




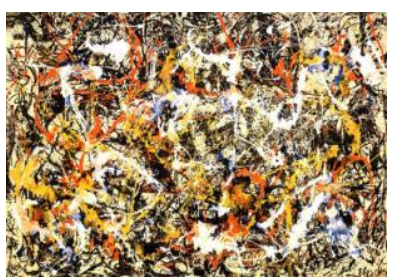

شكل (8)

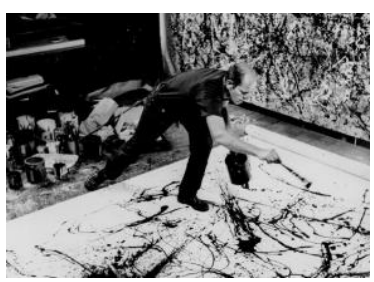

شكل (7)

2. 2.

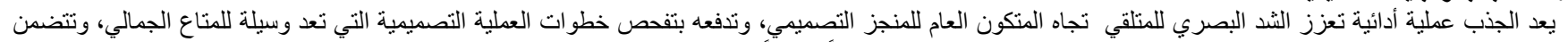

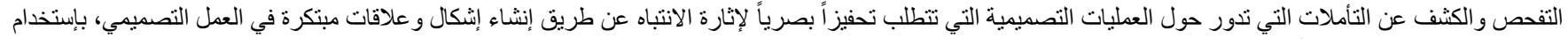

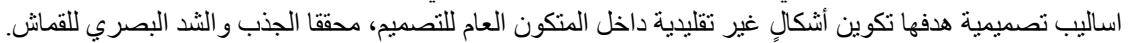

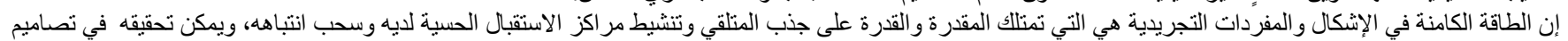

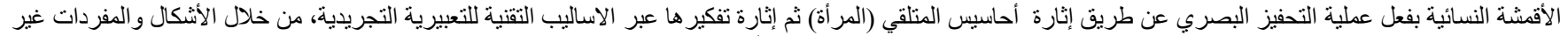

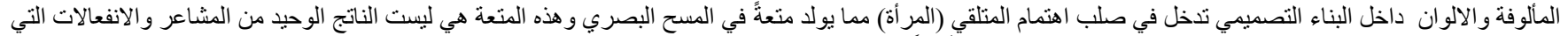

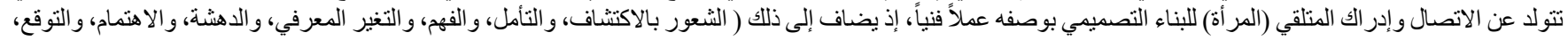

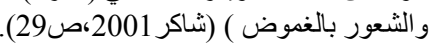

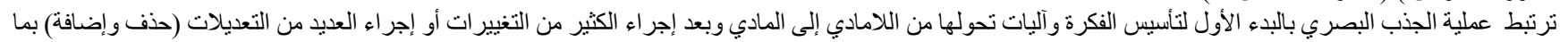

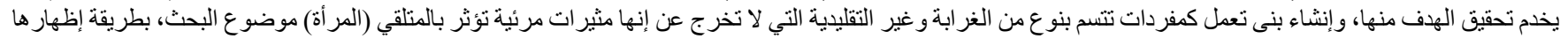

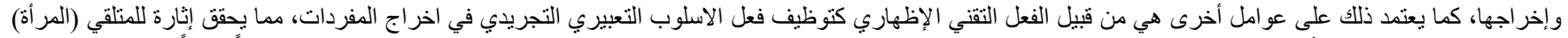

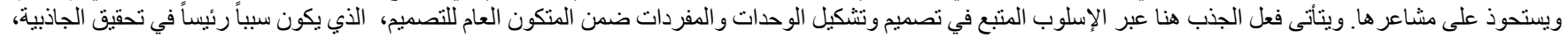

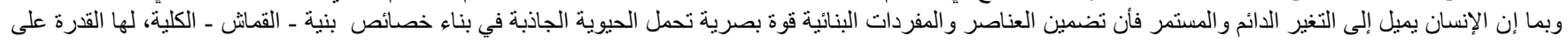

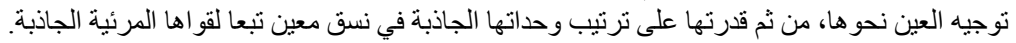

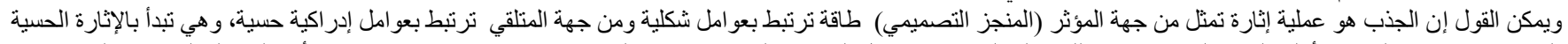

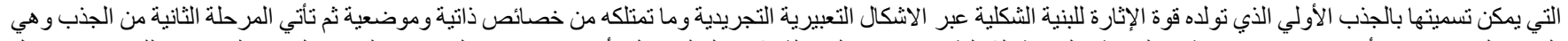

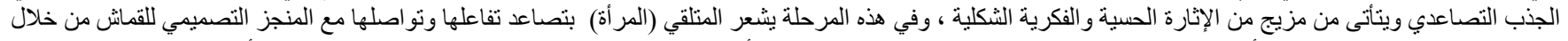

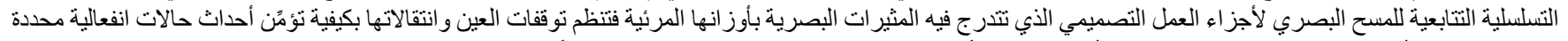

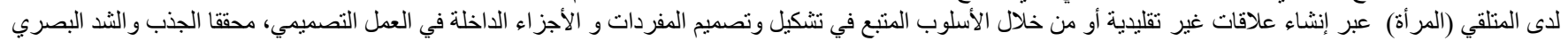
للعمل الفني التصميمي.

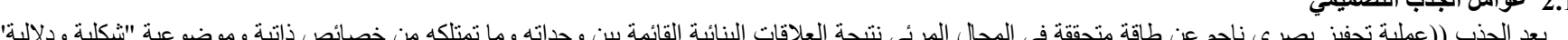

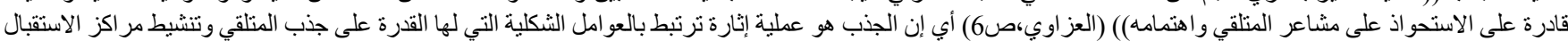

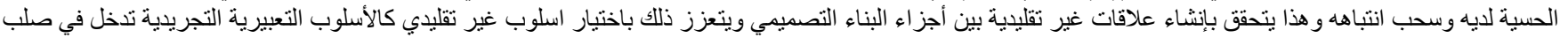

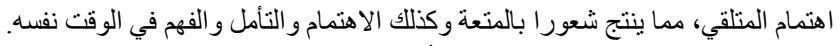

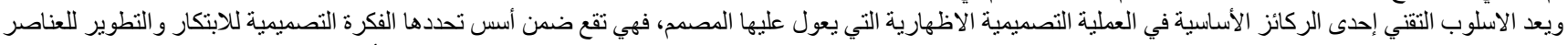

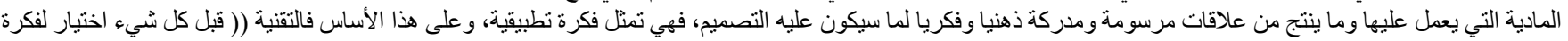

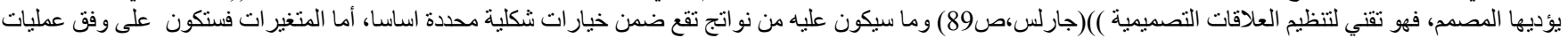

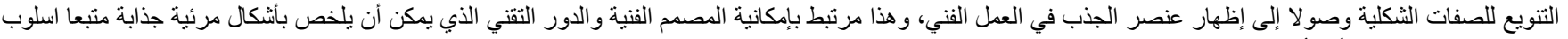

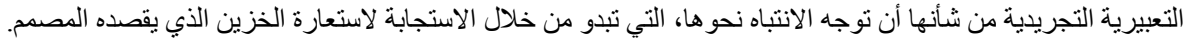

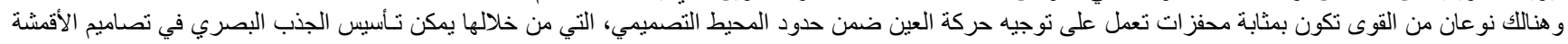

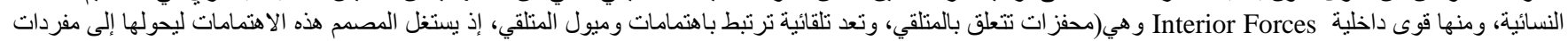

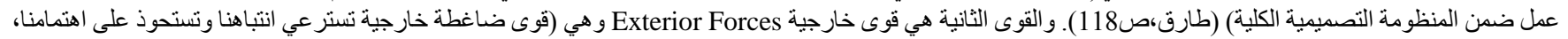

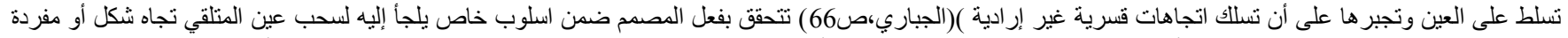

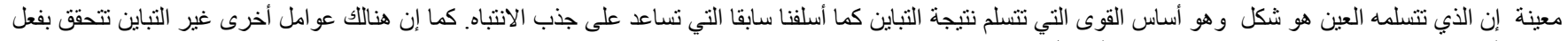

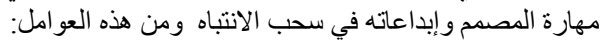

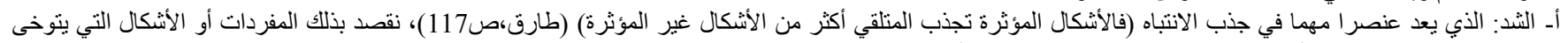

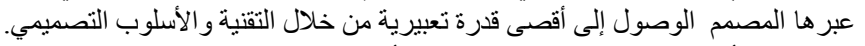

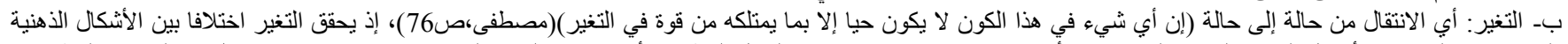

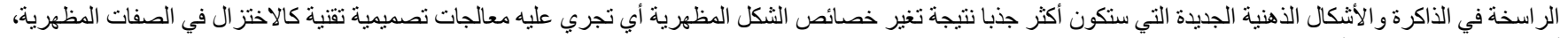
أي تغير ات شكلية جزئية أو كلية.

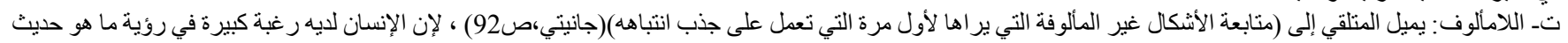

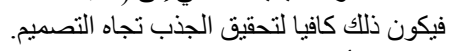

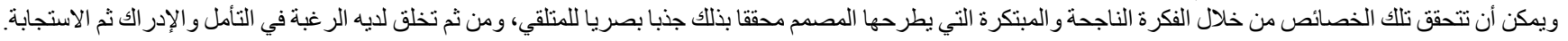

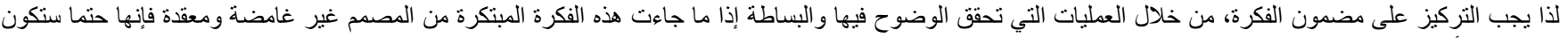

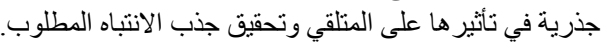




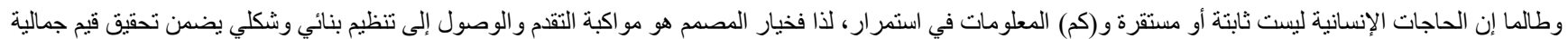

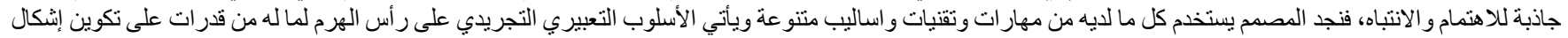

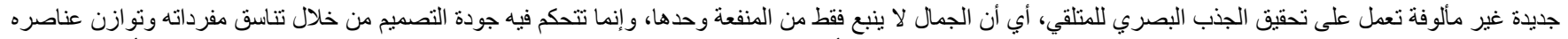

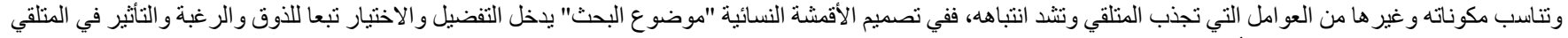

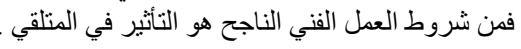

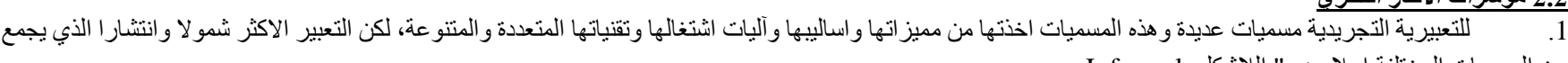

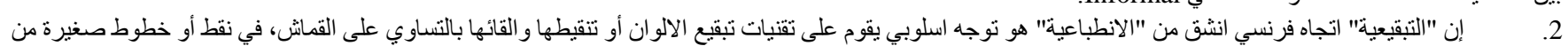

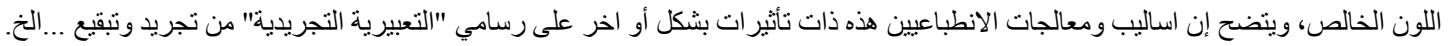

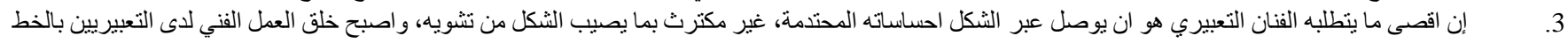
و اللون فقط دون ان تحدد الاشكال بدقة.

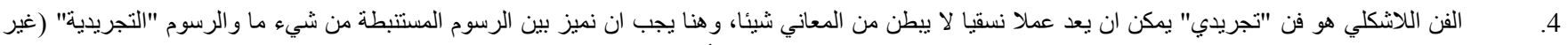

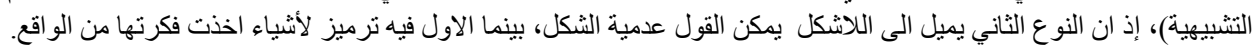

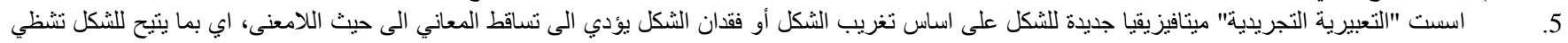

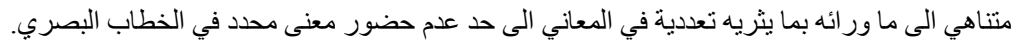

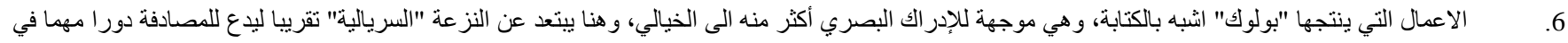
انتاج اعماله الفنية.

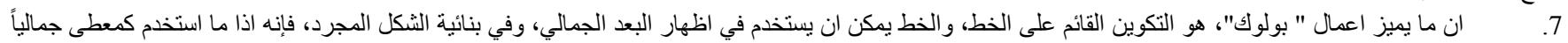

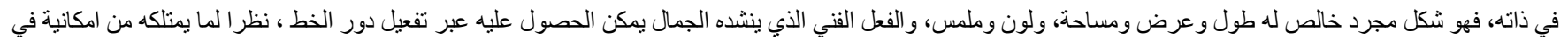

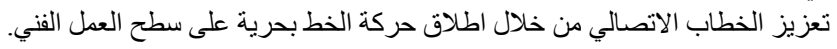

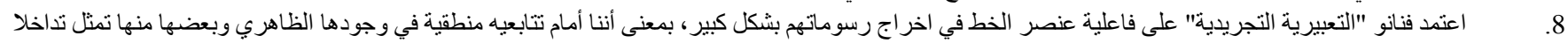

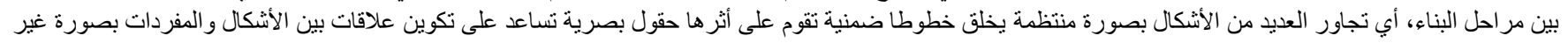

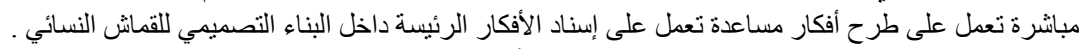

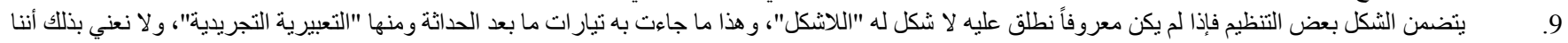

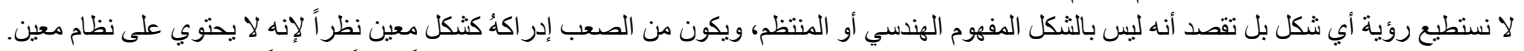

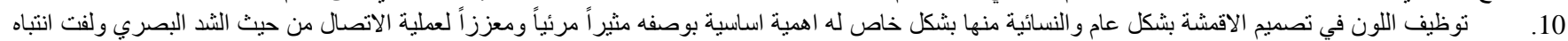

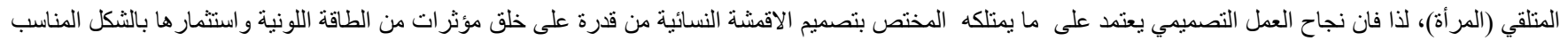

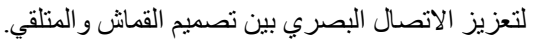

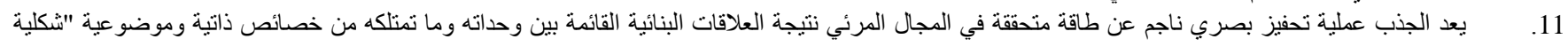
ودلالية" قادرة على جذب انتباه المتلقي واهتمامه.

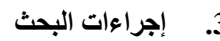

$$
\begin{aligned}
& \text { بيضضمن هذا ألفصل الإجر اءات التي اتبعها الباحث للوصول إلى أهداف البحث وكما يأتي: }
\end{aligned}
$$

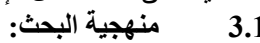

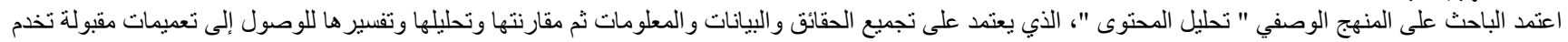

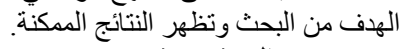

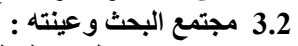

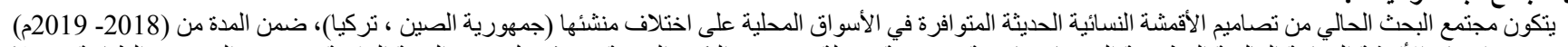

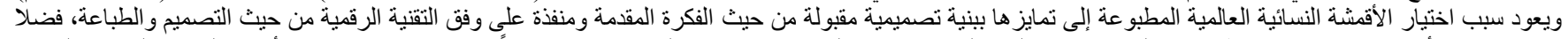

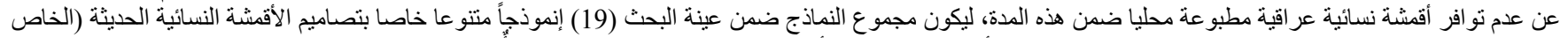

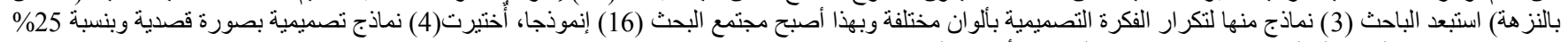

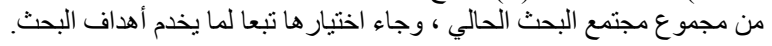

3.3 أداة البحث:

التحقيق الوصول إلى أهداف البحث أعدّت استمارة تحديد محاور التحليل تضمنت المحاور الأساسية التي تناولها الإطار النظري، إذ استند الباحث في تصميمها إلى ما تمخض عنه

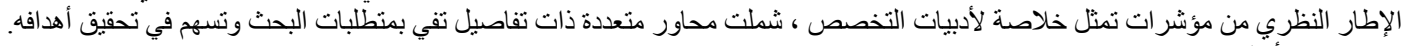

3.4 بنـاء الأداة:

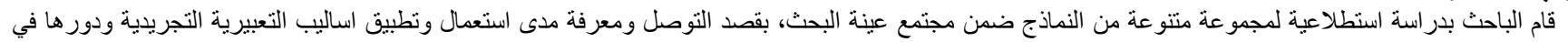

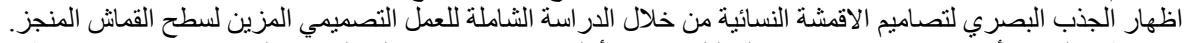

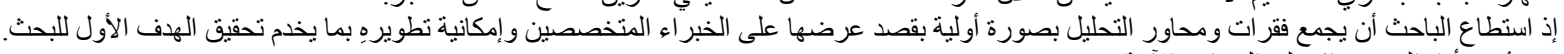

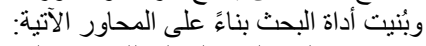

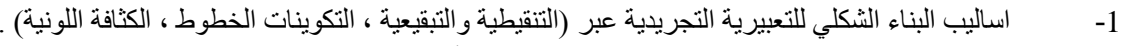

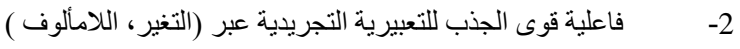

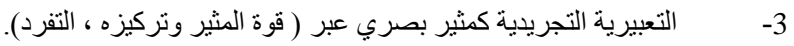




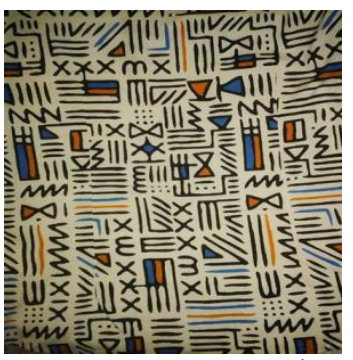

نموذج رقم (1) الوصف العام

الخامة: قماش بوليستر.

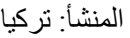

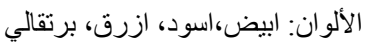

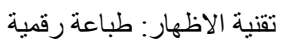
قماش محدد الوظيفة لاستخدام النزهة هلية، مكون من مفردات ورمز تعبيرية تجريدية وتجريدية هندسية.

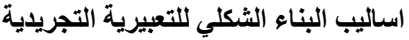

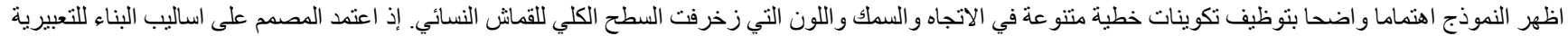

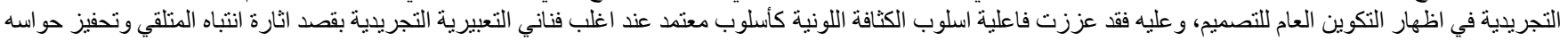

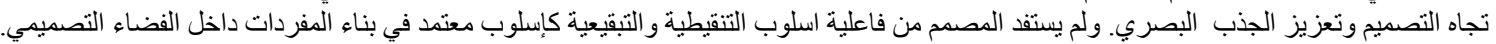

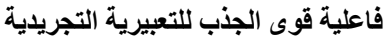

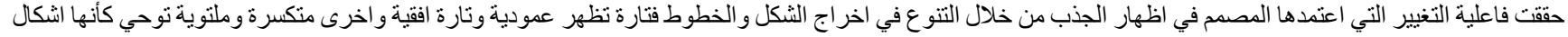

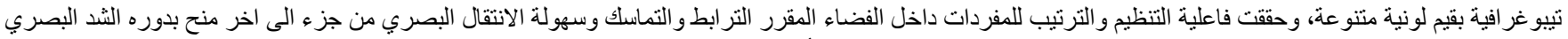

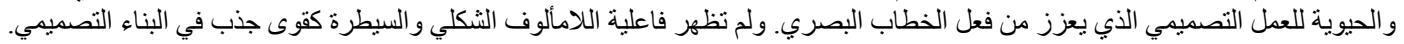

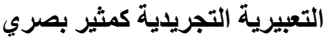

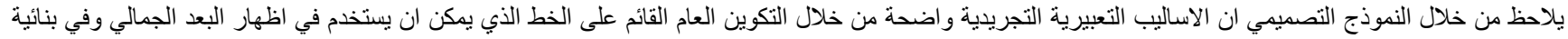

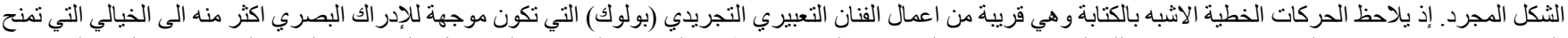

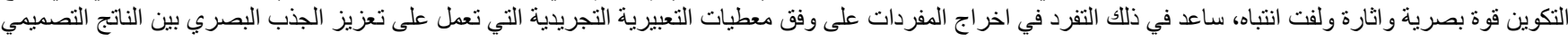

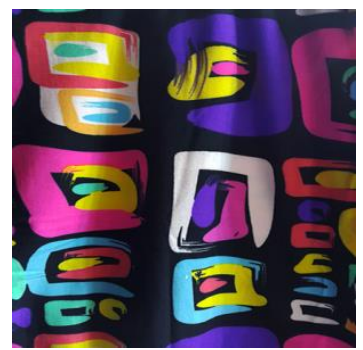

(2) نموذج رقم الومام الوصف العام الخامة: قماش بوليستر المنشأ: تركيا

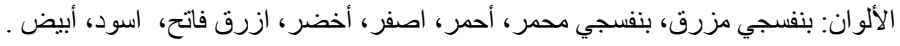
تقنية الاظهار : طباعة رقمية

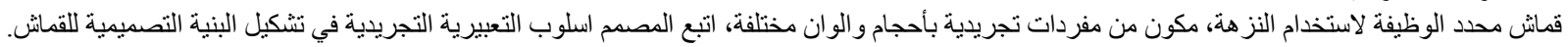

اساليب البناء الثكلي للتعبيرية التجريدية لأنية

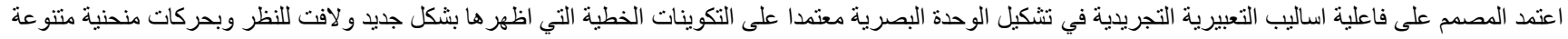

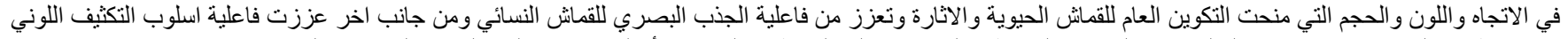

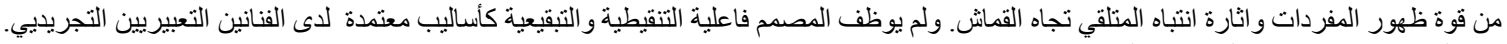
فاعلية قوى الجذب للتعبيرية التجريدية

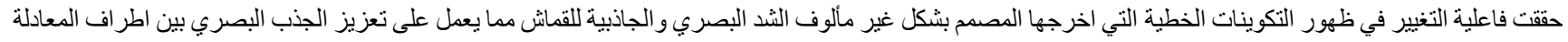

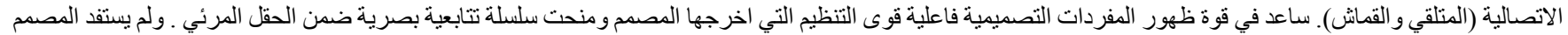
من فاعلية قوى السيطرة كقوى فاعلة في تحقيق الجذب فأب البصري. 
التعبيرية التجريدية كمثير بصري التعبري

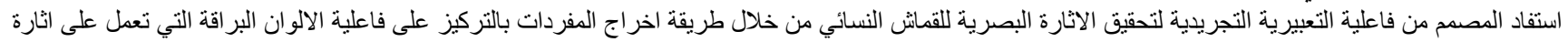

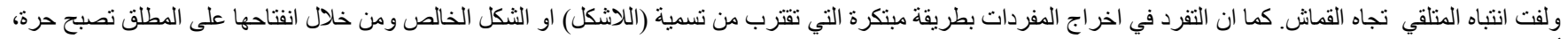

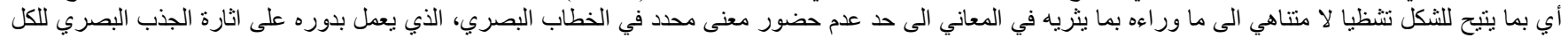
التصميمي للقماش النسائي .

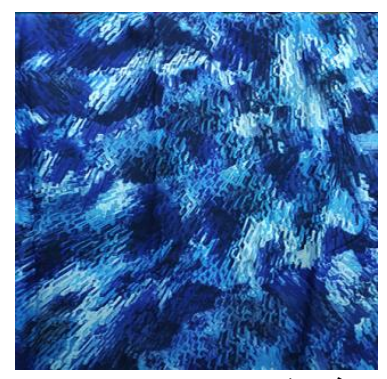

(3) نموذج رقام العمام الوصف العام الخامة: قماش بوليستر المنشأ: تركيا الألو ان: ازرق تركيا ودرجاته . تقنية الاظهار : طباعة رقمية قماش محدد الوظيفة لاستخدام النز هة ضمن مدد محددة مكون من تكوينات تعبيرية تجريدية التي حققت قيما جمالية جذابة للقماش.

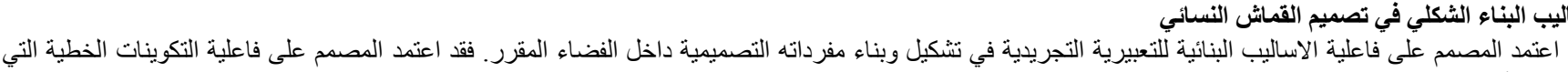

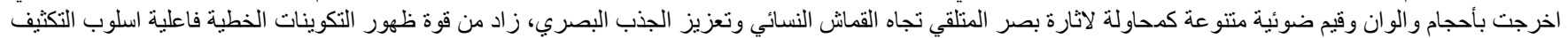

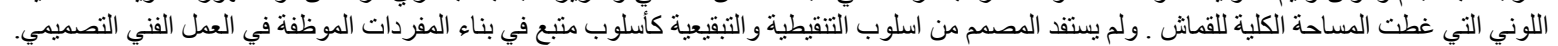

فاعلية قوى الجذب للتعبيرية التجريدية

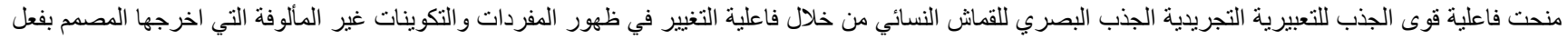

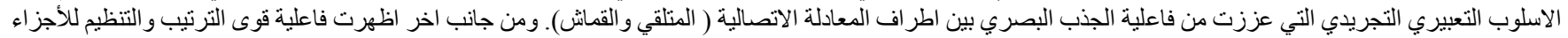

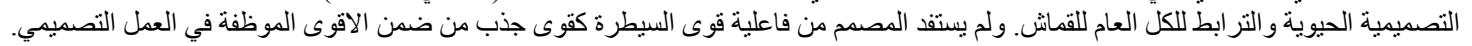

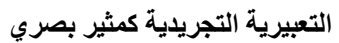

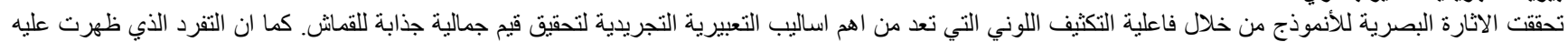
مفردات التصميم من حيث الاخر اج منحت الاثارة وسحب البصر تجاه القماث التي تعمل على تعزيز الجذب البصائ التصري للكل التصميمي.

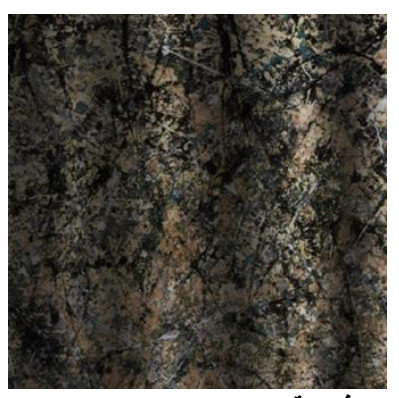

نموذج رقم (4) الوصف العام الخامة: قماش بوليستر

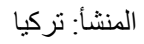

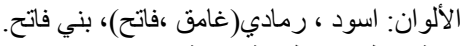
تقنية الاظهار : طباعة رقمية الوان قماش متعدد الوظيفة لاستخدام النز هة و الرسمي ضاءئ التمن مدد محددة، مكون من مفردات تجريدية، اتبع المصمم اسلوب التعبيرية التجريدية في تشكيل البنية التصميمية للقماش.

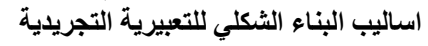

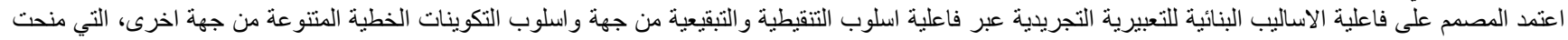

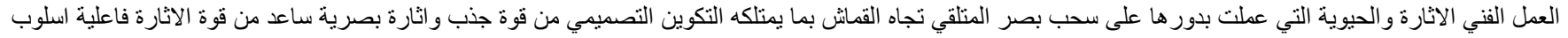

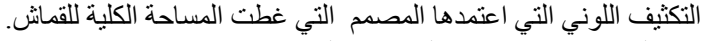
فاعلية قوى الجذب للتعبيرية التجريدية التبدية التئية

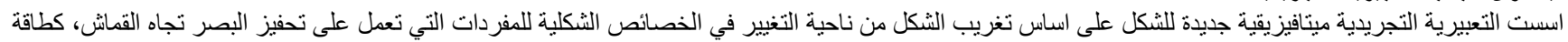

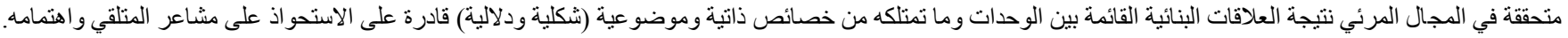




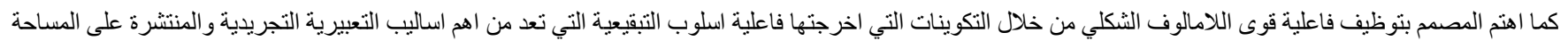

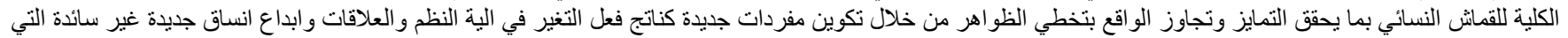

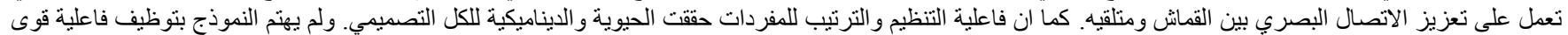

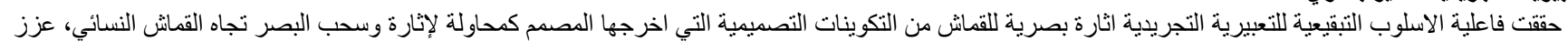

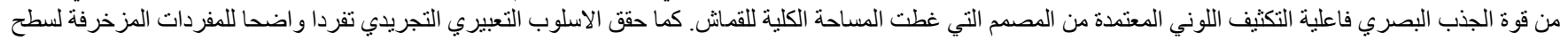
القماش و التي عززت من فاعلية الجذب البصري لطري البكرفي المعادلة الاتصالية.

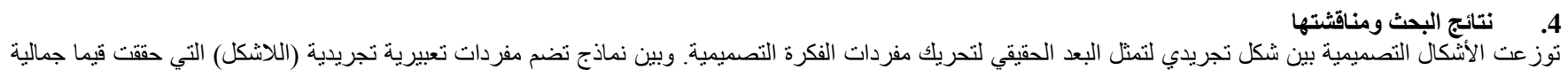

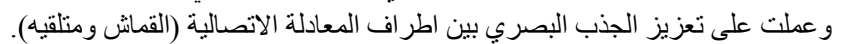

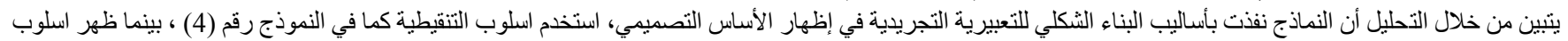

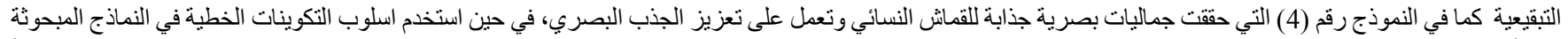

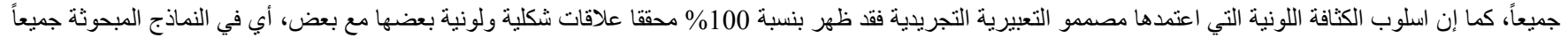

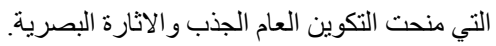

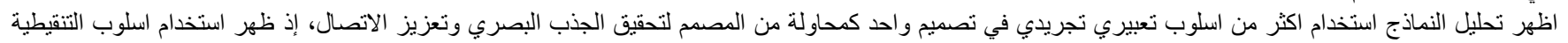

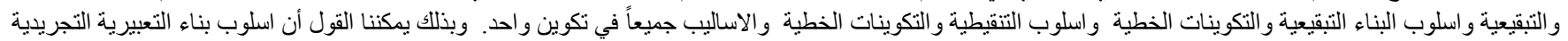

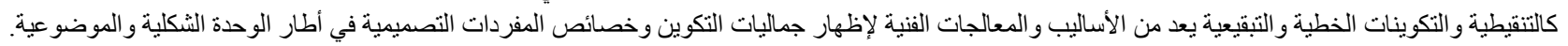

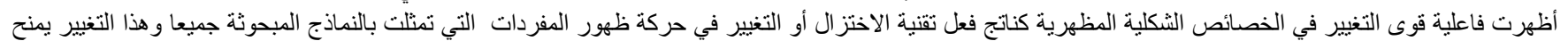

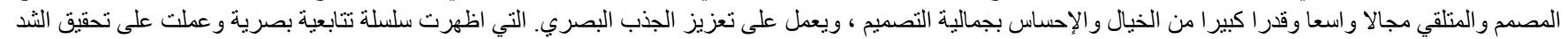

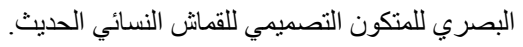

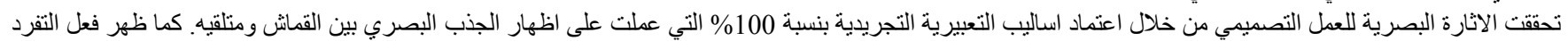

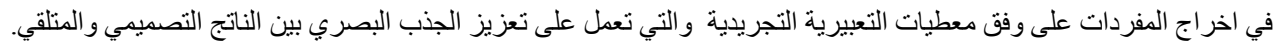

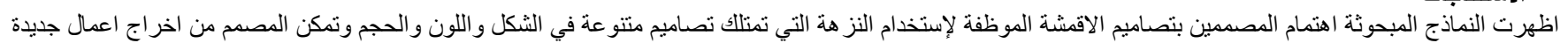

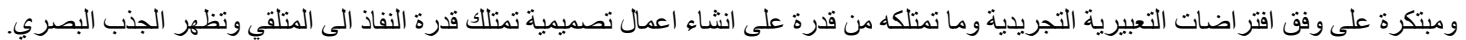

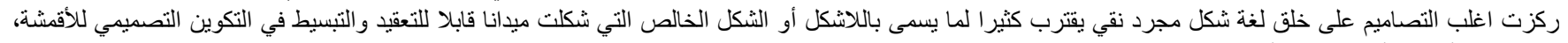

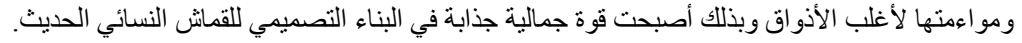

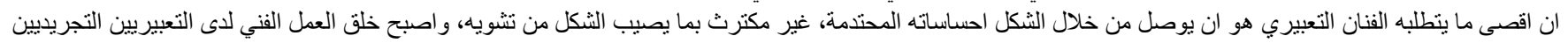

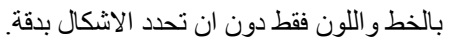

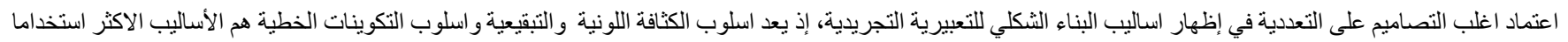

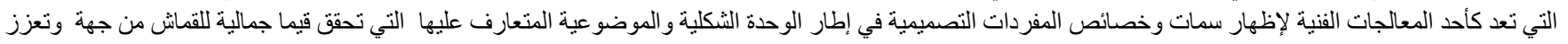

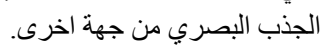

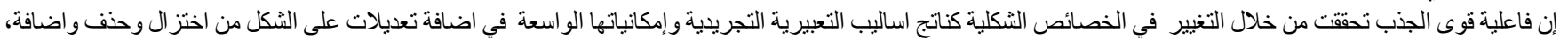

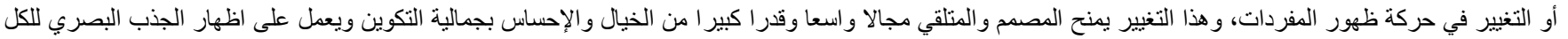

التصميمي.

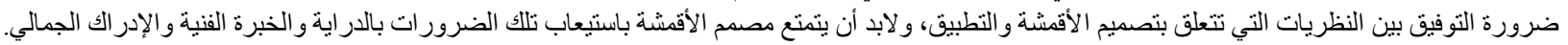

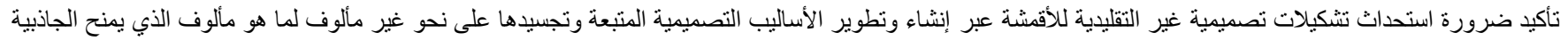

\section{References}

1- Ibn Manzour: The Tongue of the Arabs, The Egyptian Foundation for Authorship and Publishing, Vol. 3, Cairo, b

2- Ismail, Mahmoud Hassan: Principles of communication science and theories of influence, i 1, Egypt, International House for Publishing and Distribution, 20030

3- Paul Klee: Theory of Formation, I 1, T: Adel Al-Siwi, Dar Merritt, Cairo, 2003

4- Garles, Morris: Science, Art and Technology, T: Samir Abdul Rahim Al-Chalabi, No. 3, Foreign Culture, Fourth Year, Baghdad, 1984

5- Janeti, Louis de: Understanding the Cinema, T. Jaafar Ali, Al-Rasheed Publishing House, Baghdad, 1989

6- Al-Jabbari, Adib Nouri: Visual Isolation in Architecture, Ph.D. Thesis (Unpublished), University of Technology, Baghdad, 1999

7-Jiyad, Salam Jabbar: The Controversy of the Picture between Ideal Thought and Modern Painting, Ph.D. Dissertation (Unpublished),

University of Baghdad, College of Fine Arts, 2003

8- Al-Khatib, Abdullah: Mental Perception in Plastic Arts, 1st Floor, General Cultural Affairs House, Baghdad, 1998 
9- Al-Dulaimi, Riyad Hilal Mutlak: Structure of Pure Form in Modern Abstract Painting, Ph.D. Dissertation (Unpublished), University of Babylon, Faculty of Fine Arts, 2004

10- Al-Rubaie, Fakher Mohammed: The Problematic of the Absolute in Modern Painting, Ph.D. Dissertation (Unpublished), University of Babylon, Faculty of Fine Arts, 2002.

11- Sarah Neumayer: The Story of Modern Art, 2nd Edition, Ramses Younan, Contemporary Thought Series, Beirut, 2011.

12- Smith, Edward Lucy: Artistic Movements after World War II, T: Fakhri Khalil, House of Public Cultural Affairs, Baghdad, 1995.

13- Shaker Abdul Hamid: The Age of Image, Knowledge World, Kuwait, 2005.

14- Aesthetic Preference (A Study in the Psychology of Art Tasting), Knowledge World Series, No. 276, Al-Watan Press, Kuwait, 2001.

15- Tariq Ramzi, et al .: Introduction to Psychology, Presentation and Review: Mohamed Abdelkader, 1st edition, Sana'a University Publications, 1992.

16- Al-Azzawi, Hikmat Rashid Fakhri: Attractiveness in the Structure of Magazine Cover Designs (ABB Model), PhD Thesis, Baghdad University, College of Fine Arts, 2004.

17- Al-Ashry, Galal: Screams in the Face of the Age, 2nd Edition, Egypt Library Publications, Cairo, 2012.

18- Limari, Jean: Impressionism, T. Fakhri Khalil, House of Cultural Affairs, Baghdad, 1987.

19- Mahmoud Amhaz: Contemporary Art Streams, 2nd Floor, Publications Company for Distribution and Publishing, Beirut, Lebanon, 2009.

20- Contemporary Fine Art (Photography 1870-1970), Triangle House for Design, Printing and Publishing, Beirut, 1981.

21- Mustafa Ghalib: For a Philosophical Encyclopedia - Heikal, Al-Hilal House and Library, Beirut, 1982.

22- Upholstered in Language and Media: Dar Al-Sharq Publications, 7th floor, Beirut, 1984.

23- Muller, Joseph Emile: Art in the Twentieth Century, 2nd edition, T: Mahan El Khoury, Dar Atlas, Damascus, 2008.

24- Herbert Reed: Summary in the history of modern painting, i 1, T: Gloss Bakri, House of Cultural Affairs, Baghdad, 1989.

25- John, A. Walker: Art Since Pop, London, Thames and Hudson, 1975. 